\title{
Elevated Risk of Suicidal Ideation in HIV-Positive Persons
}

\author{
L. Schlebusch ${ }^{1}$ and R. D. Govender ${ }^{2}$ \\ ${ }^{1}$ Department of Behavioural Medicine, Nelson R Mandela School of Medicine, University of KwaZulu-Natal, Durban, South Africa \\ ${ }^{2}$ Department of Family Medicine, Nelson R Mandela School of Medicine, University of KwaZulu-Natal, Durban, South Africa
}

Correspondence should be addressed to L. Schlebusch; schlebuschl@ukzn.ac.za

Received 29 June 2015; Accepted 10 September 2015

Academic Editor: Verinder Sharma

Copyright (c) 2015 L. Schlebusch and R. D. Govender. This is an open access article distributed under the Creative Commons Attribution License, which permits unrestricted use, distribution, and reproduction in any medium, provided the original work is properly cited.

\begin{abstract}
Globally, suicide and HIV/AIDS remain two of the greatest healthcare issues, particularly in low- and middle-income countries. Several studies have observed a relationship between suicidal behaviour and HIV/AIDS. Materials and Methods. The main objective of this research was to determine the prevalence of elevated risk of suicidal ideation in HIV-positive persons immediately following voluntary HIV counselling and testing (VCT). The study sample consisted of adult volunteers attending the VCT clinic at a university-affiliated, general state hospital. Participants completed a sociodemographic questionnaire, Beck's Hopeless Scale, and Beck's Depression Inventory. Results. A significantly elevated risk of suicidal ideation was found in $83.1 \%$ of the patients who tested seropositive. Despite a wide age range in the cohort studied, the majority of patients with suicidal ideation were males in the younger age group (age $<30$ years), consistent with the age-related spread of the disease and an increase in suicidal behaviour in younger people. Relevant associated variables are discussed. Conclusion. The results serve as important markers that could alert healthcare professionals to underlying suicide risks in HIV-positive patients. It is recommended that screening for elevated risk of suicidal ideation and prevention of suicidal behaviour should form a routine aspect of comprehensive patient care at VCT clinics.
\end{abstract}

\section{Introduction}

Suicidal behaviour and HIV/AIDS continue to present major public health challenges especially in low- and middleincome countries [1-4]. Although there are wide variations between and within countries and subregions, research has shown that under certain circumstances HIV/AIDS patients can be at high risk for suicidal behaviour [5-9]. According to the World Health Organization (WHO), in the past few decades, global suicide rates have increased by $60 \%$ with nearly one million people worldwide dying each year as a consequence of suicide which is likely to increase to predicted suicide mortality rates of 1.53 million per annum by the year 2020 [1]. Likewise, global HIV/AIDS trends show that the number of people living with HIV/AIDS rose from around 8 million in 1990 to 34 million by the end of 2010, with more than 25 million people dying from it since it was first diagnosed in 1981 with sub-Saharan Africa carrying the greatest burden of the epidemic and Southern Africa being the most heavily affected (up to $68 \%$ of all HIVpositive people) $[7,10,11]$. South Africa has been estimated to have one of the world's largest populations of people living with HIV/AIDS (5.7 million) $[4,10,12]$. Durban is a large harbour city with one of the busiest ports in Africa and is situated on the eastern seaboard of the country in the Province of KwaZulu-Natal (where the research reported in the present paper was conducted). Some $60 \%$ of the province's economic activity takes place in this port city where $88.6 \%$ of its inhabitants are black South Africans and $68 \%$ are of working age (16-65 years), but up to one-third or more are unemployed [13]. This geographical region bears not only one of the highest HIV-seropositive prevalence rates, but also high comparative suicidal ideation rates in the general population as found in a community survey component of a large WHO international multisite study conducted in nine cities on five continents $[1,14]$. Although the results of that study showed a remarkable disparity across the sites where the research was done (illustrating how careful one must be when making national, international, and/or regional comparisons), the proportion of subjects in the general population in Durban that admitted to having had suicidal thoughts in their lifetime was $25.4 \%$. Country wide, South 
African suicide rates covering different years are equally alarming with mean annual estimates ranging from 10.9 to as high as 25 per 100,000 of the population depending on sample size and where and when the research was done [13, 15-18], but indicating a general trend that suicidal behaviour is on the increase, especially among younger people $[5,15,19]$. Several non-hospital based South African studies [15] showed that between $4 \%$ and $47 \%$ of school children surveyed expressed suicidal ideation, while in a national survey in a sample of persons aged 18 years and older the estimated lifetime prevalence rate of suicidal ideation was 9.1\% [20].

Similarly, research on suicidal ideation in HIV/AIDS patients has produced diverse results. Suicidal behaviour stems from a complex constellation of components and how they interact with the suicidal process varying from one individual to another and specific risk factors [2, 5, 21]. Not only is suicidal behaviour a complex process that ranges in degree of severity from thinking about killing oneself (i.e., suicidal ideation) to actually doing it $[2,5]$, but also in atrisk persons with HIV/AIDS the pattern and/or prevalence of suicidal behaviour may differ throughout the progression of the disease from HIV infection up to the development of full-blown AIDS and thereafter [5, 7]. HIV/AIDS carries several unique stressors $[5,7,22]$. When patients are first diagnosed to be HIV-positive, many react with disbelief and anxiety and express fear of what lies ahead. As early as 1995, a South African study showed that $17 \%$ of youth had attempted suicide because of an AIDS phobia [23]. Some studies suggest that certain patients attempt suicide within the first three months of notification of their positive status, whereas others do so within the first year [7, 24-26]. Others may become increasingly suicidal upon a deterioration of their medical condition during the advanced stages of the disease and the development of neurocognitive impairment or as a result of psychiatric complications [7, 26-29]. Higher rates of suicidal ideation in HIV-positive individuals generally have been described whereas some studies have found that some asymptomatic HIV-infected persons had higher rates than persons with AIDS or reported suicidal ideation in patients within the first week of testing for HIV [6, 9, 25, 30-32]. A study [24] of HIV-positive nonpsychiatric individuals found that two-thirds reported lifetime and one-third current suicidal ideation. In line with several studies [24-26, 29-32] from other countries that reported on the nexus between lifetime and current suicidal ideation in different HIV seropositive patient samples, a recent South African study reported high levels of suicidal ideation (27.5\%) closely linked to suicide plans among HIV-positive pregnant women [33].

However, although various statistics are available on the prevalence of suicidal ideation in HIV-positive persons, there is limited research data on the elevated risk of suicidal ideation immediately following a positive HIV diagnosis at clinics offering voluntary HIV counselling and testing (VCT), especially in developing societies such as those found in South Africa. Consequently, the purpose of the present research was to assess such risk in this cohort of patients. VCT offers patients a chance to comprehend their HIV status and, depending on the results of testing, affords them the opportunity to immediately access treatment and ultimately presents scope for them to positively alter suicidal risk behaviour, if present. This paper emanates from and is based on the results of a larger study that has produced several publications $[8,9,34-36]$ where the theoretical framework underpinning the research was, in part, to assess the psychological impact of a negative life event, that is, immediately after receiving a positive HIV test result and the resultant cascade of emotions, including those that trigger elevated risk of suicidal ideation.

\section{Methodology}

Sample recruitment consisted of 190 adult volunteer patients referred to the VCT clinic at a university-affiliated, general state hospital in the port city of Durban (mentioned earlier), South Africa, over a three-month period. One patient dropped out because of incomplete results, leaving 189 (99.5\%) of the originally recruited number of whom 157 (83.1\%) tested HIV-positive. They then voluntarily signed an informed consent form and received extensive pre- and posttest counselling according to individual patient requirements. The instruments used to assess the prevalence of suicidal ideation in recently diagnosed HIV-positive persons were a sociodemographic questionnaire, the Beck Hopelessness Scale (BHS) [37], and the Beck Depression Inventory (BDI) [38]. In accordance with previous research [9,34], we used a cut-off score of 9 and above on the BHS to identify suicidal ideation in this study. For the BDI, we used the standard cut-off scores, namely, 0-9 (minimal depression), 10-18 (mild depression), 19-29 (moderate depression), and 30-63 (severe depression). Participants completed the questionnaires at 72 hours and again at 6 weeks following VCT. SPSS software (version 15.0; SPSS Inc., Chicago, Illinois, USA) was used for data analysis, along with Pearson's chi-squared test, $t$ tests, and a binary logistic regression analysis of the sociodemographic variables. The latter used a backward stepwise method with entry and exit probabilities set at 0.05 and 0.1 based on likelihood ratio tests. The sociodemographic variables were entered into the model at step one. After five steps, the final model was reported with odds ratios (ORs) and $95 \%$ confidence intervals (CIs). In addition, to ascertain the sensitivity and specificity of cut-off points on the BDI to predict suicidal ideation, receiver operating characteristic (ROC) analyses were utilised. It yielded a summary measure, which is the area under the curve (AUC), which was considered as the probability of correct prediction [34].

Ethical approval was obtained from the University of KwaZulu-Natal (UKZN) Biomedical Research and Ethics Committee and the provincial Department of Health. The study participants were advised of the study either in English or isiZulu (the major local language spoken) as requested. All research participants' rights (including that to confidentiality) were maintained and respected. In terms of data management and storage, all electronic databases were passwordprotected and the raw data were kept in a lockable cabinet, to which only the researchers had access. If the researcher suspected that a participant was at high risk for suicide, then the relevant referral for help was made for more intensive treatment. 


\section{Results and Discussion}

In the $83.1 \%$ of the patients who tested HIV-positive, the risk of suicidal ideation was $20.5 \%$ at 72 hours, while 6 weeks thereafter the risk had increased to $28.8 \%$. The findings represent an elevated suicidal ideation risk which was significantly associated with a diagnosis of a seropositive HIV status $(p=0.013 ; 95 \%$ CI 13.97-29.92). None of the HIV-negative patients displayed suicidal ideation after being informed of their seronegative status. The mean age was 33.49 years $(\mathrm{SD}=$ 9.449) for HIV-positive patients and 37.94 years ( $\mathrm{SD}=15.238)$ for HIV-negative patients, with an overall patient participant age range of 16-79 years in line with that of the working population of the region [13]. Of the total sample, $70.8 \%$ were females and $59.4 \%$ were unemployed individuals with the majority of patients (38.5\%) falling within the 21-30-year age category. A significant association was found between HIV test results and age, while on the binary logistic regression analysis both age and gender were significant predictors of suicidal ideation $(\mathrm{OR}=0.959, p=060)$ with males having a 1.8 times higher risk of suicidal ideation than females ( $p=0.025)$, along with a lower level of education, more significant socioeconomic pressures, and exposure to the adverse influences of traditional beliefs. These findings are consistent with other researches $[3,5,7,9,13,15,39]$ that have shown an association between female vulnerability and the roles of adverse socioeconomic correlates and unemployment and low educational status and HIV infections and suicidal behaviour in sub-Saharan Africa. In terms of traditional beliefs, although researchers [2] have commented on the potential importance of cultural variables on suicidality, it has been emphasised [5] that in developing societies more research is required on the influential role of traditional beliefs in shaping responses to health behaviour and health messages. For example, some studies [7] have found that in certain beliefs HIV/AIDS is sometimes conceptualised as a mystical force resulting in negative social discourse around it, and consequently with a diminished resistance to live with it being suggestive of a "social death" experience [7]. Despite the fact that a wide age range was represented in the cohort studied, the majority of seropositive patients with suicidal ideation fell within the younger age group (age $<30$ years), consistent with the age-related spread of the disease and the increase in suicidal behaviour in younger people $[4,13,15$, 40]. There have been recent South African reports of HIVrelated "treatment fatigue" and suicide in teenagers [40].

Apart from a host of other stressors [7, 22], it is well known that people living with HIV/AIDS can experience stigma and discrimination in certain societies $[5,7,41$, 42]. At an individual level, stigma undermines a person's identity and capacity to cope with the disease, limits the possibility of disclosure, and hinders an eventual alteration of risk behaviour. Closely linked to stigmatisation are discrimination and fear of the disclosure of an individual's HIV/AIDS status. Blame is often assigned to partners for infecting them and can result in violence, dysfunctional households, and suicidal behaviour $[5,7]$. These types of reactions inevitably can have the unfavourable outcome of rendering the epidemic "invisible" and forcing people who have contracted HIV/AIDS or those who are associated with it "to go into hiding," thereby contributing to feelings of hopelessness and depression [5, 7]. Furthermore, the manner in which each person experiences and copes with the disease can be reflected in their decision of whether or not to disclose their serostatus and their right to personal privacy and dignity. In this regard, of the HIV-positive participants in the present study, $20.5 \%$ had an elevated hopelessness score on the BHS at 72 hours after receiving their diagnosis, and $28.8 \%$ had an elevated BHS score at 6 weeks. The findings demonstrated a significant increase in feelings of hopelessness over 6 weeks. Likewise, of the HIV-positive participants, $82.8 \%$ were depressed according to their BDI scores at 72 hours after receiving their diagnosis, while $78.2 \%$ had a score within the depressed range at 6 weeks. Importantly, many international and South African studies $[2,5]$ have shown that both hopelessness and depression correlate with an increased risk in suicidal behaviour, which according to our findings also apply to HIV-positive patients seen at a VCT clinic. Item 9 of BDI is represented by options of thoughts of killing oneself, to carrying or not carrying them out. A significant association was found between the responses to this item and hopelessness as defined by the BHS cut-off scores, with $p$ values of 0.036 and 0.008 at 72 hours and at 6 weeks, respectively, further confirming elevated suicidal ideation in the HIV-positive patients studied. In addition, the AUC of the ROC curve of the BDI results used to predict suicidal ideation was $0.757(p<0.001)$ at 72 hours and 0.788 $(p<0.001)$ at 6 weeks, indicating that the relevant BDI item was a good predictor of suicidal ideation. These results are supported by previous studies that found a correlation between hopelessness, depression, and suicidal ideation [5, $38,43,44]$.

Our findings have been invaluable in establishing baseline data on elevated suicidal risk in HIV-positive persons in order to implement appropriate suicide preventive intervention immediately following a positive HIV diagnosis. We have shown that this would be the most opportune time to administer such an intervention, that is, particularly within the first 72 hours after conveyance of a positive HIV diagnosis [36]. In our view, these initial hours can be considered as the "golden hours of suicide prevention" when diagnosing HIV positivity, since patients who are often lost to follow-up would still have the benefit of suicide intervention, particularly within the context of a developing society.

\section{Conclusions}

The relationship between HIV/AIDS and elevated risk of suicidal ideation has, in the past, been underresearched in developing countries. Despite the diverse findings about correlations between suicidal behaviour and HIV/AIDS, there is compelling evidence to justify screening for risk of elevated suicidal ideation and subsequent suicide risk and intervening as early as possible $[36,45,46]$, especially following notification of a positive HIV test result immediately after HIV counselling and testing. Suicide risk in other potentially life-threatening medical conditions can be complicated and 
has been explored extensively [4], for example, in cancer [47]. In the case of HIV/AIDS, many studies in different countries $[5,48]$ have shown that suicidality appears to be prevalent in some individuals but that it tends to vary according to the stage of the disease. Nevertheless, it has been shown that screening for suicide, even among highrisk populations, ultimately does translate into preventing suicides and that effective suicide prevention includes early recognition and assessment of risk, immediate response, resource referrals, and follow-up management and treatment of at-risk individuals $[2,5,35,36]$.

In the resource-limited context of developing societies, suicide risk assessment and interventions at VCT clinics are hampered by a shortage of adequately trained healthcare professionals, suicide risk screening in general, and guidelines for suicide preventive interventions. HIV counsellors are typically responsible for pre- and posttest HIV counselling and psychosocial education, and they can easily be taskshifted to screen the subjects for suicide risk and provide suicide intervention strategies. It is recommended that this should become routine as part of comprehensive care at VCT clinics. At a reasonable cost and with minimal training, this could see the effective reduction of suicidal ideation and ultimate suicide.

\section{Study Limitations}

The sample size was not large and the overall study was confined to the immediate post-HIV test period and screening of the target population being urban-based. Certain other variables which were not measured in the study could have affected the outcome. For example, there was no information on preexisting psychiatric disorders, previous suicide attempts, or a family history of suicide, and in this regard more in-depth exploratory analyses are required.

Researchers agree that suicidal behaviour is a multifaceted and complex phenomenon. The same applies to suicidal ideation. Thus, it would have been useful to also have utilised other more comprehensive measures specifically designed to assess suicidal ideation. In addition, we had access to data at two points in time, namely, 72 hours and six weeks after diagnosis. It could have been informative to have looked at three specific groups, one which started off not suicidal, a second that started off suicidal but then had their suicidal ideations resolved, and a third group that remained suicidal throughout. It is consequently important to gain greater clarity concerning the length of time that an individual may be deemed to be at elevated risk for suicidal ideation and behaviour following a traumatic event such as being diagnosed to be HIV-positive. Longitudinal studies are, therefore, needed to determine the effectiveness of screening for elevated risk of suicidal ideation in HIVinfected persons at VCT clinics over longer follow-up time periods. This would enable differentiation between variables that are more prevalent at different stages of the disease, as well as the impact of the introduction of ARVs on suicidal ideation.

\section{Conflict of Interests}

The authors declare that there is no conflict of interests regarding the publication of this paper.

\section{Authors' Contribution}

Professor Schlebusch and Dr. Govender contributed to designing and conducting the study. Dr. Govender collected the data and did the primary interpretation of them. Professor Schlebusch drafted the paper and both authors have assisted in preparation of the paper and have read and approved the content of it and are accountable for all aspects of the work.

\section{Acknowledgments}

The authors wish to thank Prenisha Pillay for her research assistance. Dr. R. D. Govender was supported by the Columbia University-Southern African Fogarty AIDS International Training and Research Programme (AITRP) funded by the Fogarty International Center, National Institute of Health (Grant no. D43TW00231).

\section{References}

[1] J. M. Bertolote, A. Fleischmann, D. de Leo, and D. Wasserman, "Suicidal thoughts, suicide plans and attempts in the general population on different continents," in Oxford Textbook of Suicidology and Suicide Prevention. A Global Perspective, D. Wasserman and C. Wasserman, Eds., pp. 99-104, Oxford University Press, Oxford, UK, 2009.

[2] D. Wasserman and C. Wasserman, Eds., The Oxford Textbook of Suicidology and Suicide Prevention. A Global Perspective, Oxford University Press, Oxford, UK, 2009.

[3] World Health Organization (WHO), Preventing Suicide. A Global Imperative: Executive Summary, 2014, http://apps.who .int/iris/bitstream/10665/131056/1/9789241564779_eng.pdf?

[4] UNAIDS, “AIDS Epidemic Update: 2012," http://www.unaids .org/sites/default/files/media_asset/20121120_UNAIDS_Global _Report_2012_with_annexes_en_1.pdf.

[5] L. Schlebusch, Suicidal Behaviour in South Africa, University of Kwa-Zulu Natal Press, Pietermaritzburg, South Africa, 2005.

[6] L. Schlebusch, "HIV/Aids og risikoen for selvmordsatferd," Suicidologi, vol. 11, pp. 30-32, 2006.

[7] L. Schlebusch and N. Vawda, "HIV-infection as a self-reported risk factor for attempted suicide in South Africa," African Journal of Psychiatry, vol. 13, no. 4, pp. 280-283, 2010.

[8] L. Schlebusch and R. D. Govender, "Age, gender and suicidal ideation following voluntary HIV counseling and testing," International Journal of Environmental Research and Public Health, vol. 9, no. 2, pp. 521-530, 2012.

[9] R. D. Govender and L. Schlebusch, "Suicidal ideation in seropositive patients seen at a South African HIV voluntary counselling and testing clinic," African Journal of Psychiatry, vol. 15, no. 2, pp. 94-98, 2012.

[10] AVERT, “Worldwide HIV \& AIDS Statistics," 2012, http://www .avert.org/worldwide-hiv-aids-statistics.htm.

[11] World Health Organization (WHO), Public Health Action for the Prevention of Suicide: A Framework, 2012, http://apps.who .int/iris/handle/10665/75166\#sthash.M1QZS3xg.dpuf. 
[12] UNAIDS, “AIDS Epidemic Update: 2007," http://data.unaids .org/pub/EPISlides/2007/2007_epiupdate_en.pdf.

[13] S. S. Naidoo and L. Schlebusch, "Sociodemographic characteristics of persons committing suicide in Durban, South Africa: 2006-2007," African Journal of Primary Health Care and Family Medicine, vol. 6, no. 1, pp. 1-7, 2014.

[14] J. M. Bertolote, A. Fleischmann, D. de Leo et al., "Suicide attempts, plans, and ideation in culturally diverse sites: the WHO SUPRE-MISS community survey," Psychological Medicine, vol. 35, no. 10, pp. 1457-1465, 2005.

[15] L. Schlebusch, "Suicidal behaviour," in Crime, Violence and Injury in South Africa: Enabling Child Safety, A. van Niekerk, S. Suffla, M. Seedat, and K. Ratele, Eds., pp. 178-194, Medical Research Council-University of South Africa, Tygerberg, South Africa, 2012.

[16] S. Burrows, L. Schlebusch, S. Musisi, E. Kinyanda, and R. Kizza, "Self-directed violence," in Violence and Health in the WHO African Region, O. Kobusingye, B. Bowman, S. Burrows, R. Matzopoulos, and A. Butchart, Eds., pp. 119-161, Regional Office for Africa, World Health Organization, Brazzaville, Congo, 2011.

[17] J. Bantjes and A. Kagee, "Epidemiology of suicide in South Africa: setting an agenda for future research," South African Journal of Psychology, vol. 43, no. 2, pp. 238-251, 2013.

[18] B. Mars, S. Burrows, H. Hjelmeland, and D. Gunnell, "Suicidal behaviour across the African continent: a review of the literature," BMC Public Health, vol. 14, article 606, 2014.

[19] S. C. S. Naidoo and L. Schlebusch, "Sociodemographic and clinical profiles of suicidal patients requiring admission to hospitals south of Durban," South African Family Practice, vol. 55, no. 4, pp. 373-379, 2013.

[20] S. Joe, D. J. Stein, S. Seedat, A. Herman, and D. R. Williams, "Non-fatal suicidal behavior among South Africans," Social Psychiatry and Psychiatric Epidemiology, vol. 43, no. 6, pp. 454461, 2008 .

[21] L. Schlebusch, "Depression and suicidal behaviour," South African Family Practice, vol. 47, no. 5, pp. 61-63, 2005.

[22] L. Schlebusch, "Suicidal behaviour in the chronic medically ill," in Suicide from a Global Perspective: Vulnerable Populations and Controversies, A. Shrivastava, M. Kimbrell, and D. Lester, Eds., pp. 65-71, Nova Science Publishers, New York, NY, USA, 2012.

[23] T. Mhlongo and K. Peltzer, "Parasuicide among youth in a general hospital in South Africa," Curationis, vol. 22, no. 2, pp. 72-76, 1999.

[24] K. Robertson, T. D. Parsons, C. Van Der Horst, and C. Hall, "Thoughts of death and suicidal ideation in nonpsychiatric human immunodeficiency virus seropositive individuals," Death Studies, vol. 30, no. 5, pp. 455-469, 2006.

[25] A. W. Carrico, M. O. Johnson, S. F. Morin et al., "Correlates of suicidal ideation among HIV-positive persons," AIDS, vol. 21, no. 9, pp. 1199-1203, 2007.

[26] E. Kinyanda, S. Hoskins, J. Nakku, S. Nawaz, and V. Patel, “The prevalence and characteristics of suicidality in HIV/AIDS as seen in an African population in Entebbe district, Uganda," BMC Psychiatry, vol. 12, article 63, 2012.

[27] W. A. Lishman, Organic Psychiatry. The Psychological Consequences of Cerebral Disorder, Blackwell, Oxford, UK, 3rd edition, 2005.

[28] B. O. Olley, S. Seedat, D. G. Nei, and D. J. Stein, "Predictors of major depression in recently diagnosed patients with HIV/AIDS in South Africa," AIDS Patient Care and STDs, vol. 18 , no. 8 , pp. $481-487,2004$.
[29] J. Badiee, D. J. Moore, J. H. Atkinson et al., "Lifetime suicidal ideation and attempt are common among HIV+ individuals," Journal of Affective Disorders, vol. 136, no. 3, pp. 993-999, 2012.

[30] B. Kelly, B. Raphael, F. Judd et al., "Suicidal ideation, suicide attempts, and HIV infection," Psychosomatics, vol. 39, no. 5, pp. 405-415, 1998.

[31] S. Perry, L. Jacobsberg, and B. Fishman, "Suicidal ideation and HIV testing," The Journal of the American Medical Association, vol. 263, no. 5, pp. 679-682, 1990.

[32] N. A. Cooperman and J. M. Simoni, "Suicidal ideation and attempted suicide among women living with HIV/AIDS," Journal of Behavioral Medicine, vol. 28, no. 2, pp. 149-156, 2005.

[33] T. J. Rochat, R. M. Bland, M. Tomlinson, and A. Stein, "Suicide ideation, depression and HIV among pregnant women in rural South Africa," Health, vol. 5, no. 3, pp. 650-661, 2013.

[34] R. D. Govender and L. Schlebusch, "Hopelessness, depression and suicidal ideation in HIV-positive persons," South African Journal of Psychiatry, vol. 18, no. 1, pp. 16-21, 2012.

[35] R. D. Govender and L. Schlebusch, "A suicide risk screening scale for HIV-infected persons in the immediate post-diagnosis period," Southern African Journal of HIV Medicine, vol. 14, no. 2, pp. 58-63, 2013.

[36] R. D. Govender, L. Schlebusch, and T. Esterhuizen, "Brief suicide preventive intervention in newly diagnosed HIV-positive persons," African Journal of Psychiatry, vol. 17, no. 2, pp. 543547,2014

[37] A. T. Beck, A. Weissman, D. Lester, and L. Trexler, "The measurement of pessimism: the Hopelessness scale," Journal of Consulting and Clinical Psychology, vol. 42, no. 6, pp. 861-865, 1974.

[38] A. T. Beck, R. A. Steer, J. S. Beck, and C. F. Newman, "Hopelessness, depression, suicidal ideation, and clinical diagnosis of depression," Suicide and Life-Threatening Behavior, vol. 23, no. 2, pp. 139-145, 1993.

[39] J. R. Hargreaves, C. P. Bonell, T. Boler et al., "Systematic review exploring time trends in the association between educational attainment and risk of HIV infection in sub-Saharan Africa," AIDS, vol. 22, no. 3, pp. 403-414, 2008.

[40] K. Child, “Treatment fatigue killing kids," The Times, vol. 3, 2014, http://www.timeslive.co.za/.

[41] K. Child, "HIV gays still 'outcasts"' The Times, vol. 6, 2014, http://www.timeslive.co.za/.

[42] B. Maughan-Brown and N. Spaull, "HIV-related discrimination among grade six students in nine Southern African countries," PLoS ONE, vol. 9, no. 8, Article ID e102981, 2014.

[43] J. A. Chiles, K. D. Strosahl, Z. Y. Ping et al., "Depression, hopelessness, and suicidal behavior in Chinese and American psychiatric patients," The American Journal of Psychiatry, vol. 146, no. 3, pp. 339-344, 1989.

[44] M. J. Priester and G. A. Clum, "Attributional style as a diathesis in predicting depression, hopelessness, and suicide ideation in college students," Journal of Psychopathology and Behavioral Assessment, vol. 14, no. 2, pp. 111-122, 1992.

[45] J. Catalan, R. Harding, E. Sibley, C. Clucas, N. Croome, and L. Sherr, "HIV infection and mental health: suicidal behavioursystematic review," Psychology, Health \& Medicine, vol. 16, no. 5, pp. 588-611, 2011.

[46] A. W. Carrico, "Elevated suicide rate among HIV-positive persons despite benefits of antiretroviral therapy: implications for a stress and coping model of suicide," American Journal of Psychiatry, vol. 167, no. 2, pp. 117-119, 2010. 
[47] L. Schlebusch, "Suicide risk and cancer," in Medical Conditions Associated with Suicide Risk, L. Berman and M. Pompili, Eds., pp. 59-74, American Association of Suicidology, Washington, DC, USA, 2011.

[48] N. A. Cooperman, "Suicide risk and cancer," in Medical Conditions Associated with Suicide Risk, L. Berman and M. Pompili, Eds., pp. 159-174, American Association of Suicidology, Washington, DC, USA, 2011. 


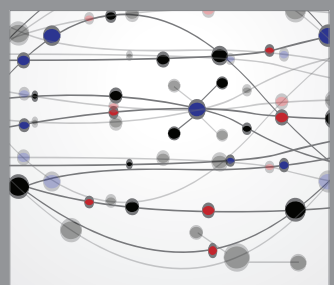

The Scientific World Journal
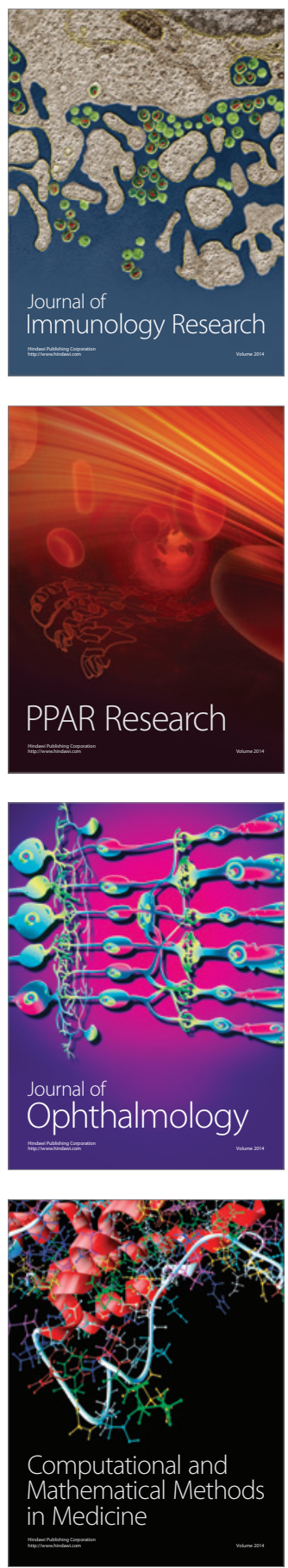

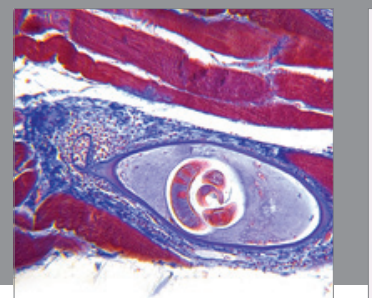

Gastroenterology

Research and Practice
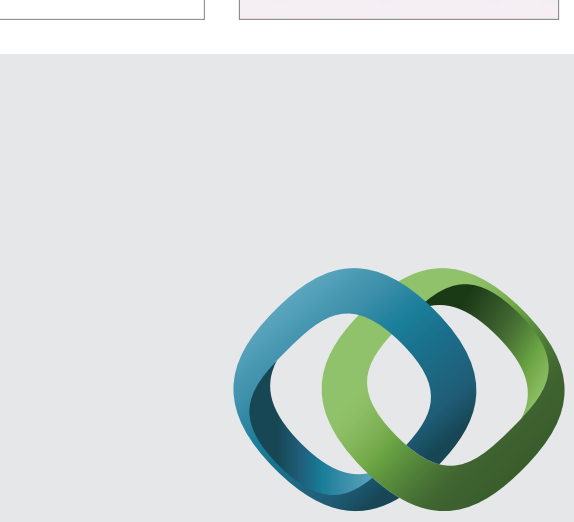

\section{Hindawi}

Submit your manuscripts at

http://www.hindawi.com
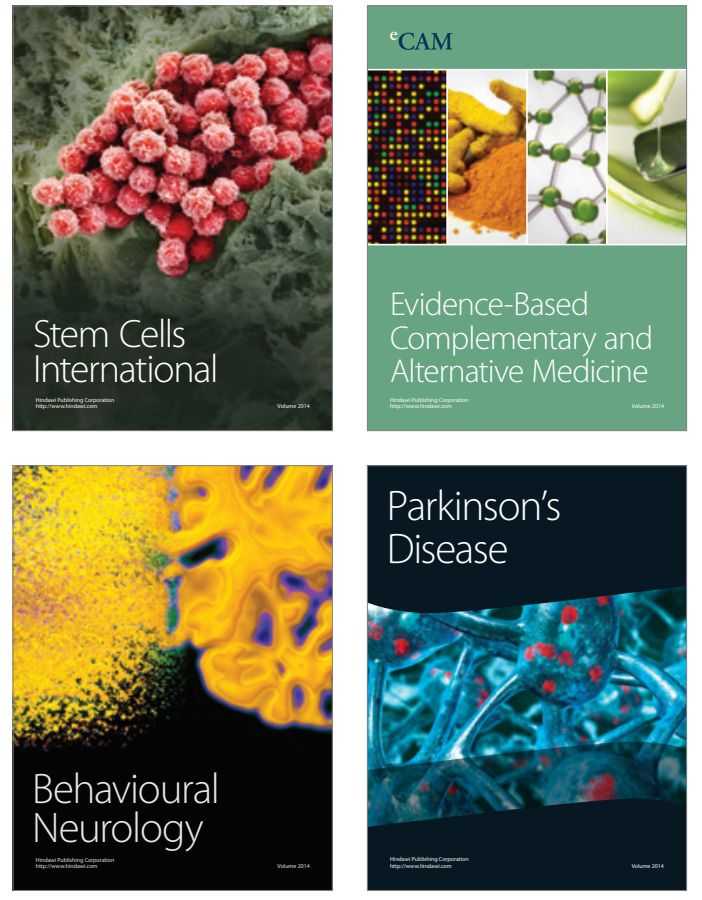
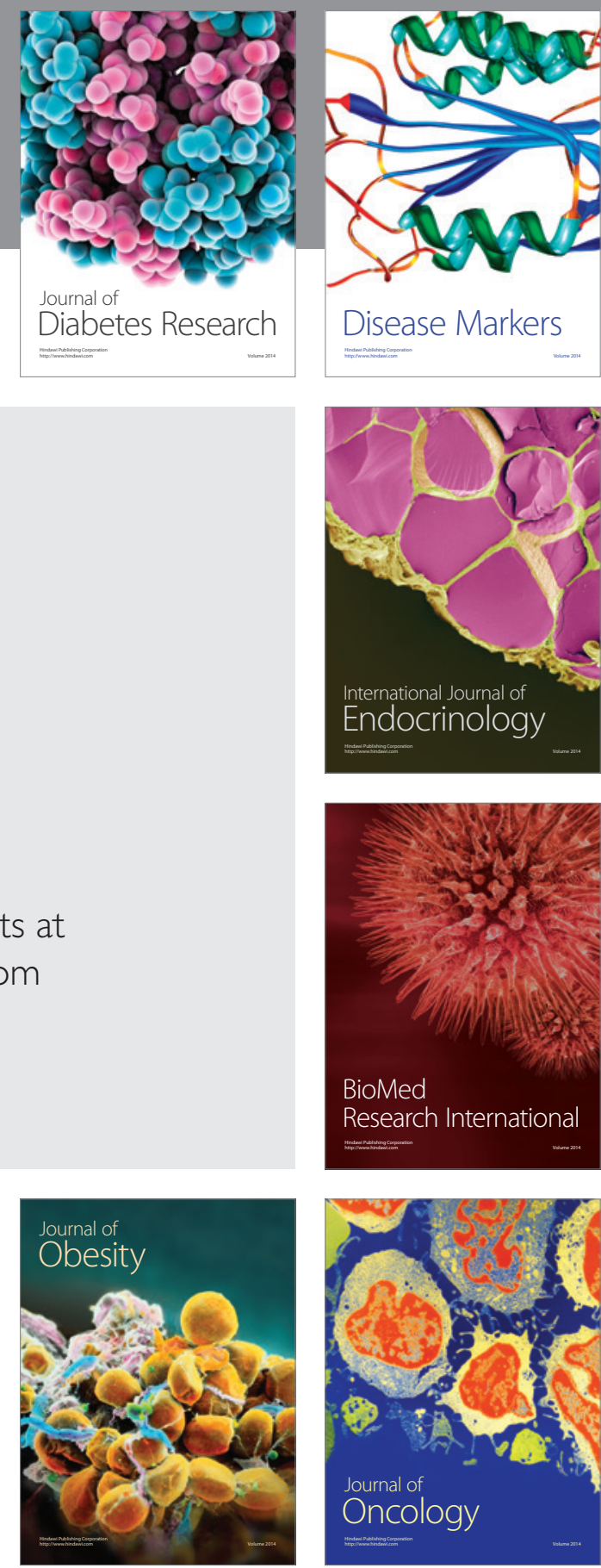

Disease Markers
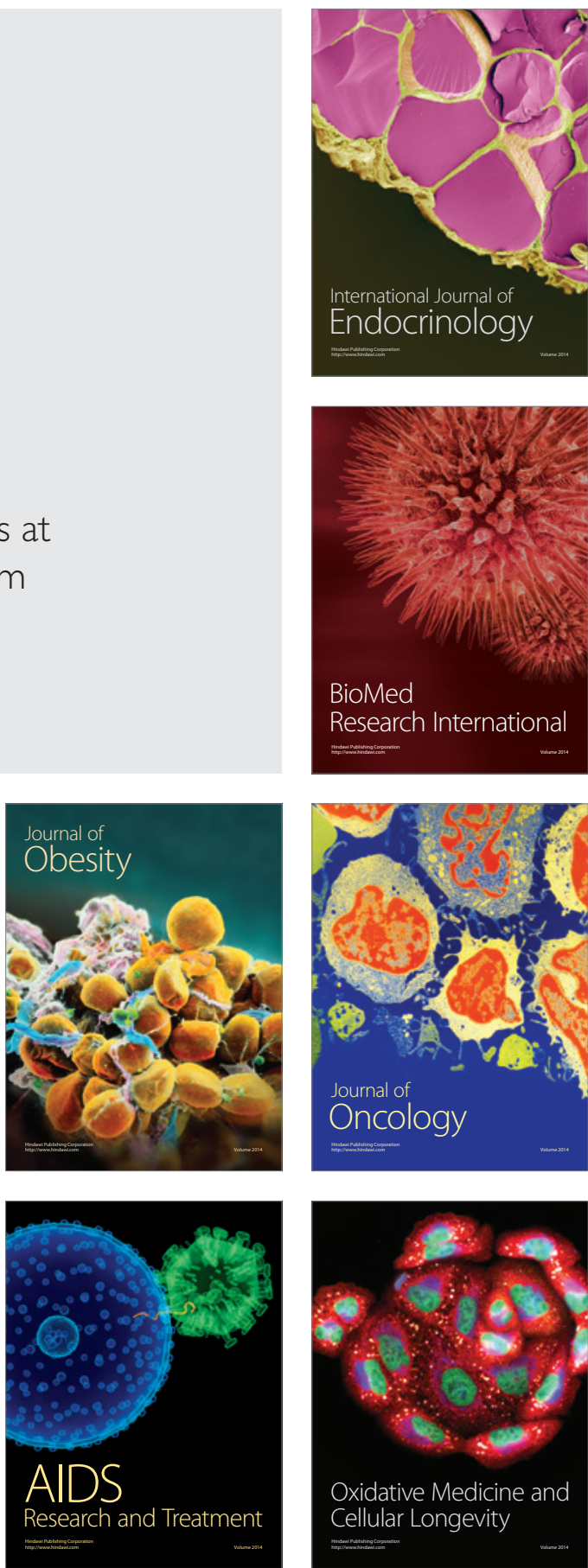